\title{
Development and Psychometric Properties of an Assessment for Persons With Intellectual Disability-The interRAI ID
}

\author{
Lynn Martin ${ }^{\star \dagger}$, John P. Hirdes ${ }^{\star \dagger}$, Brant E. Fries ${ }^{\ddagger}$, and Trevor F. Smith* \\ ${ }^{\star}$ Department of Health Studies and Gerontology, University of Waterloo, Waterloo; ${ }^{\dagger}$ Home Research Institute, Waterloo, ON, Canada; \\ and ${ }^{\ddagger}$ Institute of Gerontology and School of Public Health, University of Michigan and Ann Arbor VA Medical Center, Ann Arbor, MI, \\ USA
}

\begin{abstract}
This paper describes the development of the interRAI-Intellectual Disability (interRAI ID), a comprehensive instrument that assesses all key domains of interest to service providers relative to a person with an intellectual disability (ID). The authors report on the reliability and validity of embedded scales for cognition, self-care, aggression, and depression. Four provider agencies volunteered to participate and assessed a total of 160 community-dwelling adults with ID using the interRAI ID, Dementia Questionnaire for Persons with Mental Retardation, and Reiss Screen for Maladaptive Behavior. All scales had acceptable levels of internal consistency (Cronbach's alpha 0.74-0.93) and good relationships with the criterion measures $(r=0.50-0.93, p<0.0001)$. The development of the interRAI ID represents an important and successful first step toward an integrated, comprehensive, and standardized assessment of adults with ID. Use of this instrument may lead to more appropriate support planning, enhanced communication between various professionals supporting persons with ID, and a more seamless approach to supports across the health and social service systems.
\end{abstract}

Keywords: comprehensive assessment, intellectual disability, interRAI, reliability, validity

To ensure that adequate support and services are provided to adults with an intellectual disability (ID), an improved understanding of their strengths, preferences, and needs is essential. As there are multiple functional impairments, behavioral problems, psychiatric illnesses, and medical conditions affecting persons with ID, this population often receives services from both the health and social services sectors. For this reason, the use of a standardized comprehensive assessment system that covered all key life areas of interest to service providers in either sector would be of undeniable value.

interRAI is an international not-for-profit research organization consisting of approximately 50 members from 26 countries, and has developed a "suite" (http://www.interrai.org) of integrated, comprehensive assessment instruments that are designed to evaluate the needs of persons in different care settings (Hirdes et al., 1999), including long-term care, home care, inpatient psy-

Received January 20, 2006; accepted October 16, 2006

Correspondence: Lynn Martin, PhD, Department of Health Studies and Gerontology, University of Waterloo and Homewood Research Institute, 150 Delhi Street, Guelph, ON, Canada N1E 6K9. Tel: +1 519824 1010;

Fax: +1 519767 3571; E-mail: lrmartin@healthy.uwaterloo.ca chiatry, emergency psychiatry, community mental health, acute care hospitals, rehabilitation, palliative care, and assisted living. While the instruments focus on the needs and issues specific to the population of interest, they were designed to be compatible with one another (i.e., common "core" set of items, assessment language, and methods for data collection) to enhance communication between providers, to facilitate the tracking of individuals and outcomes throughout the system, and to promote a more seamless approach to service across sectors.

In Canada, the Ontario Ministry of Health and Long-Term Care mandated the use of interRAI instruments in long-term care, home care, and inpatient psychiatry. This has allowed for clearer communication between those sectors and has promoted a more integrated and seamless approach to health care. InterRAI systems have similarly been adopted in other nations around the world.

The interRAI-Intellectual Disability (interRAI ID) represents the latest addition to the interRAI suite of instruments, and was designed for adults with ID. This paper reports on the psychometric properties of the interRAI ID. Specifically, it examines the internal consistency and criterion validity of embedded measures for cognition, self-care, aggression, and depression, as well as the convergent validity of the embedded measure of cognition. 


\section{METHOD}

\section{Participants}

The participants were 160 adults (aged 18 years or more) who had been diagnosed with ID at birth or in early childhood (i.e., not as a result of traumatic brain injury) and were receiving residential services from four community-based provider agencies in Ontario. Participants (or their substitute decision-makers) provided informed consent prior to taking part in the study (response rate $=97.6 \%$ ). All assessments were completed between May and November, 2003.

Staff in each of the agencies $(n=19$ developmental services workers) received a 1-day training session on the completion of the interRAI ID and chosen validation scales, and were also given a Users' Manual for the interRAI ID. The intent, definition, suggested process to obtain the information, and coding for every item in the instrument, is detailed in the manual (Hirdes et al., 2003).

Staff were encouraged to use their professional judgement and to use all sources of information available to complete the interRAI ID. In particular, assessors were told to rely on their own observations, to speak with the person being assessed as well as his or her family members and friends (if available), to consult with other staff or professionals involved in supporting the individual, as well as to access all available documentation. The first two interRAI ID assessments of all assessors were reviewed by the research team's educator, who also provided ongoing clinical support to all assessors for the duration of the study.

\section{Secondary Analyses}

The internal consistency of embedded interRAI scales among persons with ID will also be reported using: (1) census data on all adults with ID who received chronic care hospital services between 1996 and 2003 and assessed with interRAI's instrument for long-term care-the RAI $2.0(n=753)$; and (2) data from the pilot implementation of the now mandated interRAI instrument for mental health-the RAI-MH $(n=129)$.

\section{Measures}

InterRAI ID The interRAI ID contains 391 items designed to assess the status of persons with all levels of ID across all areas of life (Table 1). As the instrument intends to screen for a broad range of needs or problems, only the minimum number of items needed to identify a potential problem is included for each area. When such a problem is identified, the assessor relies on a combination of his or her professional skills, the individual's wishes, and resource availability to decide on whether and how the problem should be addressed. The assessment is designed to take approximately 1 hour to complete, though the first few will take
TABLE 1

Domain content in the interRAI ID

\begin{tabular}{lc}
\hline \hline & \\
Domains & Number of items \\
\hline Personal information & 65 \\
Health service history & 5 \\
Cognition & 15 \\
Communication & 8 \\
Hearing and vision & 4 \\
Physical functioning and self-care & 34 \\
Physical health & 55 \\
Medications & 5 \\
Skin condition & 7 \\
Oral and nutritional status & 24 \\
Psychiatric diagnoses & 6 \\
Mental state indicators & 34 \\
Life events & 14 \\
Behavior & 15 \\
Psychosocial well-being and social supports & 33 \\
Education & 1 \\
Vocation & 8 \\
Recreation & 20 \\
Prevention and intervention & 31 \\
Home environment & 7 \\
\hline
\end{tabular}

interRAI ID = interRAI-Intellectual Disability.

longer. InterRAI's experience in other sectors shows that the time to complete decreases as the assessor becomes more familiar with the item content (usually after five or six assessments).

In addition to gathering administrative information and identifying various areas of strength, preference, and need, the interRAI ID also collects information useful for measuring individual outcomes, evaluating quality of services provided, determining the resource intensity of service needs (case-mix).

A body of evidence exists that describes the reliability and validity testing of interRAI instruments. Both the inter-rater reliability of items and internal consistency of embedded scales are reported to be in the good to excellent range (Mor, 2004; Morris, Carpenter, Berg, \& Jones, 2000; Morris et al., 1997). Both the inter-rater reliability of items and internal consistency of embedded scales are reported to be in the good to excellent range (Mor, 2004; Morris, Carpenter, Berg, \& Jones, 2000; Morris et al., 1997).

The internal consistency for each of the life areas represented in the instrument may not be calculated, as they are comprised of items with varying response options that are not intended to be summed to produce a global score. For example, within the cognition section, items on memory are assessed as yes (memory problem) or no (memory OK), the person's performance in decision-making is rated according to level of independence $(0=$ Independent, 1 = Modified independence, $2=$ Minimally impaired, 3 = Moderately impaired, $4=$ Severely impaired, $5=$ No discernible consciousness), and the potential for delirium 
is assessed by asking whether specific behaviors were absent in the last 3 days (scores $=0$ ), present in the last 3 days but not of recent onset (score $=1$ ), or present in the last 3 days and differing from usual functioning (score $=2$ ). These items are not summed to produce a cognition score; rather, an algorithm based on decision-making and short-term memory, in addition to items on expressive communication (in the communication section) and self-performance in eating (in the physical functioning section) are used to derive the interRAI's measure of cognitive performance.

A series of measures are embedded in the interRAI instruments to help professionals understand the person's functioning in various areas (Morris et al., 2000). These measures-also embedded in the interRAI ID_-are useful not only for describing current functional and clinical characteristics, but are also valuable for triggering service plans, monitoring change over time, and evaluating the effects of supports or interventions. Given that these measures are currently being used in Ontario to describe the status of persons with ID receiving long-term care, home care, and inpatient psychiatric services, they were chosen for inclusion in this study. The psychometric properties for scales measuring cognition, self-care, aggression, and depression are examined here as these also represent some of the major domains of interest in this population. However, as persons with ID access healthcare services, the internal consistency of these scales will be presented using Ontario's data on the population of persons with ID receiving chronic care hospital services (assessed with interRAI's instrument for long-term care) and a sample of persons with ID receiving inpatient psychiatric services (assessed with interRAI's instrument for mental health); these results are based on the work of Martin (2004).

The Cognitive Performance Scale (CPS) is a predictive algorithm based on a decision tree that describes cognitive status. It uses four items: short-term memory, decision-making, expression, and self-performance in eating (Morris et al., 1994). The result is a 7-point scale where scores range from intact (0), borderline intact (1), mildly impaired (2), moderately impaired (3), moderately to severely impaired (4), severely impaired (5), to very severely impaired (6). Though the CPS is designed to assess the person's cognitive functioning, it can also be used over time to detect change in cognitive performance, and possibly, in combination with other measures (e.g., functional decline), to alert professionals to possible dementia. Please note that calculation of Cronbach's alpha requires that the scale under evaluation be summated; As the CPS is based on a predictive algorithm incorporating items assessing different aspects of cognition that are scored differently, its internal consistency may not be calculated.

The Activities of Daily Living Hierarchy (ADL-H) Scale is a measure of ADL performance that classifies ADLs according to the stages at which they can no longer be performed, rather than simply summing reduction in functioning (Morris, Fries, \& Morris, 1999). Based on four ADL items (i.e., personal hygiene, toilet use, mobility, and eating), an algorithm is used to compute a 7 -point scale, ranging from independent (0), to supervision (1), to limited assistance (2), to extensive assistance 1 (3) and extensive assistance 2 (4), to dependent (5), and total dependence (6). Excellent internal consistency of items in the ADL-H Scale was reported for adults with ID in psychiatric and complex continuing care hospitals (Cronbach's alpha 0.90 and 0.85 , respectively).

The Aggressive Behavior Scale (ABS) is a summated scale indicating the level of aggression exhibited over the last 3 days, based on four items: verbal abuse, physical abuse, socially inappropriate disruptive behavior, and resistance to care. Scores range between 0 and 12, where higher scores indicate higher levels of aggression. Though a weaker value was reported for adults with ID in inpatient psychiatry (Cronbach's alpha 0.59), excellent internal consistency was reported in complex continuing care (Cronbach's alpha 0.93).

The Depression Rating Scale (DRS) is a seven-item summated scale providing information on signs of possible depression, based on the presence of seven indicators over the last 3 days (negative statements, persistent anger, expressions of unrealistic fears, repetitive health complaints, repetitive anxious complaints, worried facial expression, and crying or tearfulness) (Burrows, Morris, Simon, Hirdes, \& Phillips, 2000). Scores vary between 0 and 14, and a score of 3 or more is considered to be indicative of possible depression warranting further investigation (Burrows et al., 2000). Good internal consistency was reported for adults with ID in psychiatric and complex continuing care hospitals (Cronbach's alpha 0.79 and 0.78 , respectively).

The level of ID severity (i.e., mild, moderate, severe, and profound) is also among the items in the interRAI ID. Assessors are asked to record the level of ID severity, as documented in the person's records. If only the IQ score is available in the records, assessors are asked to record the level of ID that corresponded to that score.

Criterion measures The following criteria were used to review the literature for potential criterion measures for validation of interRAI scales: (1) the instrument was designed specifically for use among adults with ID; (2) the instrument could be used with persons with varying levels of ID severity; (3) the instrument could be completed by a nonclinician (e.g., direct support staff or informal caregiver); (4) the instrument had well-established psychometric properties; and (5) the instrument was relatively simple in terms of administration, scoring, and interpretation. Based on these criteria, the Dementia Questionnaire for Persons with Mental Retardation (DMR) and Reiss Screen for Maladaptive Behavior (Reiss Screen) were chosen.

The DMR is a 50-item informant-based instrument, and each item is scored on a 3-point Likert-type scale (Evenhuis, 1995). A total of eight summated subscales and two summary scores are generated: the Sum of Cognitive Scores (SCS) and Sum of Social Scores (SOS). The SCS represents the sum of 22 items on shortand long-term memory and orientation, with scores ranging from 0 to 44; higher scores are indicative of more cognitive impairment. The SOS represents the sum of items on speech, practical skills, mood, activity and interest, and behavioral dis- 
turbance, with scores ranging from 0 to 60. Acceptable to excellent inter-rater reliability has been reported for the scales in the SCS and SOS (Evenhuis, 1995).

As scores are influenced by the presence of severe physical and psychological impairment, a diagnosis of dementia cannot be made using the DMR alone (Evenhuis, 1995); rather, cut-off points to signal the need for further investigation into possible dementia have been developed based on the person's level of ID and their SCS and SOS scores. Further, given that the diagnosis of dementia is based on presence of cognitive and functional decline, single completion of the DMR cannot alone inform on possible dementia (Aylward, Burt, Thorpe, Lai, \& Dalton, 1995). However, the SCS can inform on the person's cognitive functioning at a single point in time, and highlight decline (and possible dementia) when used longitudinally.

The Practical Skills Subscale consists of eight items measuring the individual's ability to complete everyday activities. Scale scores may range from 0 to 16 , with higher scores pointing to more problems with practical skills. Evenhuis (1995) reports excellent inter-rater reliability for this scale $(r=0.94)$.

The Reiss Screen is a 38 -item informant-based instrument designed to screen for psychopathology and problematic behavior in adults with ID (Burt \& Aylward, 2000; Reiss, 1994; Sturmey \& Bertman, 1995). Items are rated on a 3-point Likert-type scale and its simplicity ensures that it may be completed by non-mental health professionals and laypersons. Eight subscale scores may be derived in addition to a 26-item summary score, where higher scores signify an increased risk of pathology.

The Reiss Screen's assessment of depression generates two summated scores. The Behavioral Signs of Depression Scale rates the occurrence of anxiety, crying, fearfulness, oversensitivity, and sadness, whereas the Physical Signs of Depression Scale rates body stress, eating problems, low energy, regressive behavior, and sleeping problems. For each, scores may range from 0 to 10 , and cut-off scores of 5 and 4 on the Behavioral and Physical Signs of Depression subscales, respectively, are used to point to the presence of possible depression.

The Aggressive Behavior Subscale consists of five items relating to presence of aggressive behavior, destructive behavior, hostility, impulsivity, and temper tantrums. Scores may range from 0 to 10 , and a cut-off score of 5 suggests problems with aggression.

\section{Analyses}

Sample characteristics and scale scores Descriptive statistics will be used to illustrate select demographic characteristics and scale scores (interRAI, DMR, and Reiss Screen) for the study sample.

Reliability: internal consistency of interRAI scales Cronbach's alpha coefficient will be used to assess the internal consistency of embedded interRAI scales. This coefficient represents a measure of how well the items in a particular domain represent a single concept, and may only be used in cases of summated scales (i.e., where each item is rated similarly and contributes equally to the total score) (Carmines \& Zeller, 1979). Again, the CPS is based on a predictive algorithm where nonparallel items measuring different aspects of cognition contribute differently to the total score-its internal consistency cannot be calculated.

Validity: face validity, convergent validity, and criterion validity Face validity of the interRAI ID was established through a literature review and involvement of frontline workers in instrument development. Convergent validity of the CPS and DMR as global measures of cognitive status was established by testing their relationship to the person's documented level of ID. Criterion validity was established by testing the relationship between interRAI scales and the appropriate DMR and Reiss Screen scales. Given their potential use as a measure of global cognitive performance and as a screen for dementia (if used longitudinally), the relationship between the CPS and SCS was tested using the Spearman correlation coefficient. This statistic was also used to evaluate the association between the ADL-H and the Practical Skills subscales as measures of performance in self-care. Pearson product-moment correlation coefficients were used to test the relationship between DRS and the Behavioral and Physical Depression subscales, as well between the ABS and Aggression subscale.

\section{RESULTS}

\section{Sample Characteristics}

Most participants were male (59.0\%) and never married $(98.0 \%)$ with a mean age of 38.8 years $(\mathrm{SD}=12.7)$. A diagnosis of "mental retardation cause not specified" was noted for $70.6 \%$ of participants, Down syndrome for $10.0 \%$, and autism spectrum disorder (autism) for $8.1 \%$. According to their records, $17.8 \%$ had mild ID, $14.7 \%$ had moderate ID, $24.8 \%$ had severe ID, and $10.2 \%$ had profound ID. The severity of ID was not recorded in the charts of almost one-third of participants (32.5\%). Most participants lived in a group home or assisted living setting $(73.1 \%)$ and with nonrelatives (75.6\%), though some lived alone $(9.4 \%)$, with their parent(s)/guardian(s) (9.4\%), or with another relative $(5.6 \%)$. On average, participants had been clients of the community agency completing the assessment for 12.4 years $(\mathrm{SD}=9.7)$.

\section{Scale Scores}

Tables 2-4 present participants' scores for interRAI ID, DMR, and Reiss Screen scales, respectively. According to the interRAI ID, the majority of participants scored as moderately impaired for cognition (28.1\%), though CPS scores did vary by level of ID (CPS scores increased with increasing levels of ID severity). Persons in the sample tended to be independent in completing ADLs (36.3\%). Participants did not exhibit signs of clinically relevant depression (86.8\% showed no signs of depression), but did show 
TABLE 2

interRAI scale scores

\begin{tabular}{|c|c|c|}
\hline & $n$ & $\%$ \\
\hline \multicolumn{3}{|l|}{ Cognitive Performance Scale } \\
\hline Intact $(0)$ & 18 & 11.3 \\
\hline Borderline intact (1) & 26 & 16.3 \\
\hline Mild impairment (2) & 10 & 6.2 \\
\hline Moderate impairment (3) & 45 & 28.1 \\
\hline Moderate-severe impairment (4) & 13 & 8.1 \\
\hline Severe impairment (5) & 19 & 11.9 \\
\hline Very severe impairment (6) & 29 & 18.1 \\
\hline \multicolumn{3}{|l|}{ Activities of Daily Living Hierarchy } \\
\hline Independence $(0)$ & 58 & 36.3 \\
\hline Supervision (1) & 25 & 15.6 \\
\hline Limited assistance (2) & 16 & 10.0 \\
\hline Extensive assistance-level I (3) & 16 & 10.0 \\
\hline Extensive assistance-level II (4) & 6 & 3.8 \\
\hline Dependence (5) & 11 & 6.9 \\
\hline Total dependence (6) & 28 & 17.5 \\
\hline \multicolumn{3}{|l|}{ Depression Rating Scale } \\
\hline No signs of depression (0-2) & 138 & 86.8 \\
\hline Possible depression (3+) & 21 & 13.2 \\
\hline \multicolumn{3}{|l|}{ Aggressive Behavior Scale } \\
\hline No to mild aggression $(0-4)$ & 143 & 89.4 \\
\hline \multirow[t]{2}{*}{ Severe $(5+)$} & 17 & 10.6 \\
\hline & $n$ & Median \\
\hline \multicolumn{3}{|l|}{ Cognitive Performance Scale } \\
\hline Mild ID & 28 & 1.0 \\
\hline Moderate ID & 23 & 2.0 \\
\hline Severe ID & 39 & 5.0 \\
\hline Profound ID & 16 & 6.0 \\
\hline Undocumented level of ID & 51 & 3.0 \\
\hline
\end{tabular}

ID = intellectual disability.

TABLE 3

DMR scale scores

\begin{tabular}{lcc}
\hline \hline & $n$ & Mean (SD) \\
\hline Sum of Cognitive Scores & & \\
$\quad$ All & 160 & $19.1(15.3)$ \\
Mild ID & 28 & $2.7(2.6)$ \\
Moderate ID & 23 & $12.4(11.7)$ \\
Severe ID & 39 & $30.1(10.5)$ \\
Profound ID & 16 & $39.9(5.0)$ \\
Undocumented level of ID & 51 & $14.7(11.7)$ \\
DMR: practical skills scores & 160 & $4.8(6.1)$ \\
\hline
\end{tabular}

DMR $=$ Dementia Questionnaire for Persons with Mental Retardation; ID = intellectual disability.
TABLE 4

Reiss Screen scale scores

\begin{tabular}{|c|c|c|c|c|c|c|}
\hline \multirow{3}{*}{$\begin{array}{l}\text { Subscale } \\
\text { value }\end{array}$} & \multicolumn{6}{|c|}{ Subscale } \\
\hline & \multicolumn{2}{|c|}{$\begin{array}{l}\text { Behavioral } \\
\text { Signsof } \\
\text { Depression }\end{array}$} & \multicolumn{2}{|c|}{$\begin{array}{c}\text { Physical } \\
\text { Signsof } \\
\text { Depression }\end{array}$} & \multicolumn{2}{|c|}{ Aggression } \\
\hline & $n$ & $\%$ & $n$ & $\%$ & $n$ & $\%$ \\
\hline 0 & 90 & 60.0 & 77 & 50.3 & 79 & 51.6 \\
\hline 1 & 26 & 17.3 & 42 & 27.5 & 22 & 14.4 \\
\hline 2 & 178 & 11.3 & 11 & 7.2 & 21 & 13.7 \\
\hline 3 & 7 & 4.7 & 13 & 8.5 & 12 & 7.8 \\
\hline 4 & 3 & 2.0 & 10 & 6.5 & 4 & 2.6 \\
\hline $5+$ & 7 & 4.7 & - & - & 15 & 9.8 \\
\hline
\end{tabular}

TABLE 5

Internal consistency of interRAI scales

\begin{tabular}{lc}
\hline \hline & $\begin{array}{c}\text { Cronbach's alpha } \\
\text { (standardized) }\end{array}$ \\
\hline Activities of Daily Living Hierarchy Scale & 0.93 \\
Depression Rating Scale & 0.78 \\
Aggressive Behavior Scale & 0.74 \\
\hline
\end{tabular}

some signs of aggression (59.4\%). Only 10.6\% showed more severe signs of aggressive behavior. According to the DMR, overall, participants scored at the midpoint of the SCS range of scores $(X=19.1, S D=15.3)$, though results varied by level of ID. Not surprisingly, the SCS increased with increasing levels of ID severity. The average rating on the Practical Skills subscale 4.8 $(\mathrm{SD}=6.1)$, indicating higher levels of independence in the study sample. According to the Reiss Screen, study participants did not experience problems with depression (only $4.7 \%$ and $6.5 \%$, respectively, exceeded the cut-off scores for behavioral or physical signs of depression) or aggression (9.8\% exceeded the cut-off score).

\section{Reliability: Internal Consistency of interRAI Scales}

Standardized Cronbach's alpha coefficients were computed to test the internal consistency of the interRAI scales embedded in the interRAI ID (Table 5), and these were found to be internally consistent in the study sample. The ADL-H Scale had the highest level of internal consistency (alpha $=0.93)$, followed by the DRS $($ alpha $=0.78)$ and ABS $($ alpha $=0.74)$. 
L. Martin et al. • Psychometric Properties of the interRAI ID

TABLE 6

Criterion validity of interRAI scales: Spearman ${ }^{\mathrm{a}}$ and Pearson ${ }^{\mathrm{b}}$ correlations with subscales on the DMR and Reiss Screen

\begin{tabular}{lll}
\hline \hline interRAI scales & \multicolumn{1}{c}{ Validation scales } \\
\hline $\begin{array}{l}\text { Cognitive Performance Scale (CPS) } \\
\text { Activities of Daily Living Hierarchy (ADL-H) Scale } \\
\text { Depression Rating Scale (DRS) }\end{array}$ & $\begin{array}{l}\text { DMR: Sum of Cognitive Scores (SCS) } \\
\text { DMR: Practical Skills Subscale }\end{array}$ \\
Aggressive Behavior Scale (ABS) & $\begin{array}{l}\text { Reiss: Behavioral Signs of Depression } \\
\text { Reiss: Physical Signs of Depression }\end{array}$ \\
\hline
\end{tabular}

${ }^{*} P<0.0001$.

a Spearman correlation coefficients are reported for the CPS and SCS, as well as for the ADL-H and Practical Skills subscale.

${ }^{b}$ Pearson correlation coefficients are reported for the DRS and Depression subscales (Behavioral and Physical) and for the ABS and Aggression subscale.

$\mathrm{DMR}=$ Dementia Questionnaire for Persons with Mental Retardation.

\section{Validity: Convergent Validity of the CPS and SCS}

The relationship between the scores on the CPS and SCS and the level of ID severity was tested among the 106 participants that had a documented level of ID severity (three cases were missing, and 51 cases did not have a documented level of ID). The results indicated that both the CPS $(r=0.79, p<0.0001)$ and the SCS $(r=0.83, p<0.0001)$ were highly and positively associated with the level of ID severity.

\section{Validity: Criterion Validity of interRAI ID Scales}

Table 6 displays the relationship between interRAI ID scale scores and the corresponding criterion measures. Each embedded scale was adequately to highly correlated with the relevant criterion measure. The highest correlation was between the CPS and SCS $(r=0.83, p<0.0001)$, followed by the ADL-H and Practical Skills subscale $(r=0.79, p<0.0001)$, the DRS and Behavioral Signs of Depression subscale $(r=0.65, p<0.0001)$, the ABS and Aggression subscale $(r=0.60, p<0.0001)$, and the DRS and the Physical Signs of Depression subscale $(r=0.50, p<0.0001)$.

\section{DISCUSSION}

The main purpose of the interRAI ID is to provide staff with the information needed for planning supports through assessment of all life domains of importance to identify individual strengths, preferences, and needs. The instrument screens for issues in all areas of life to provide a truly holistic view of the person's needs. Staff can use the individual items to guide the planning process, as well as the embedded measures. These measures were developed to improve the applicability of the information available in the interRAI instrument by providing an easily understandable rating of the person's status in various domains.

This study demonstrated that the measures embedded in interRAI instruments are internally consistent and valid among a population of community-dwelling adults with ID. Further, the study revealed that these scales were also internally consistent among patients with ID receiving care in chronic and psychiatric care hospitals. These findings not only encourage the use of these scales to assess adults with ID in the community, they also validate their ongoing use to describe persons with ID in formal healthcare settings where other interRAI instruments have been mandated.

Given the multiple applications of interRAI instruments (i.e., service planning, assessing quality, resource utilization, and outcomes), the use of the interRAI ID may be preferable to simple combination of multiple scales. The latter method is complicated by the fact that assessment instruments will have different criteria for completion (e.g., qualifications of assessor, length of observation period), as well as by the cost associated with purchasing multiple copies of a variety of tools. It should be noted that interRAI generally provides noncommercial users (e.g., service providers, governments, and researchers) with a royalty-free license to use its instruments (http://www.interRAI.org).

Further, use of the interRAI ID in agencies supporting adults with ID would serve to facilitate communication between developmental and social service providers and formal healthcare providers in jurisdictions that have implemented one or more interRAI instruments. For example, in Ontario, developmental services staff, who have identified an individual as being at risk of depression (based on a DRS score of 3), could easily speak to mental health professionals as they are familiar with the DRS. Also, should that person be admitted to an inpatient psychiatric unit for treatment, his or her status could be compared directly with that in the community prior to admission, and assessment conducted at discharge could inform community-based staff on any special areas of need.

The interRAI ID is being used by the Ontario Ministry of Community and Social Services (MCSS) to help form the planning process for closure by 2009 of Ontario's three remaining institutions for persons with ID. The MCSS has recognized the need for compatibility with the assessments used by Ontario's Ministry of Health and Long-Term Care, as the responsibility 
for the overall support and care provided to these individuals will soon be shared by both Ministries. The interRAI ID is also being used by five community-based agencies in south-western Ontario to assess adults 50 years of age or more who are part of a study funded by the Canadian Institutes for Health Research that is seeking to examine the needs of older adults with ID.

The work to evaluate the interRAI ID contains some limitations. First, the community-dwelling sample we used may not necessarily be generalizable to adults with ID, or to all Ontarians with ID. However, demographic characteristics of the participants corresponded to the expectations of administrators at each of the participating community agencies. Further, a number of clinical relationships identified in the literature were replicated. Specifically, ADL impairment and expressive and receptive communication skills were positively correlated to the person's level of ID severity. Second, the limited number of community staff ( $n=19)$ completing the assessments in the pilot study may have biased results obtained on internal consistency. However, as the values of Cronbach's alphas reported in the pilot sample are similar to those obtained in hospital settings, the results do not appear to have been influenced by the small number of assessors. Third, more work is warranted with respect to the further development of the instrument's applications (i.e., planning, quality, and resource utilization), as well as further testing of psychometric properties (i.e., inter-rater reliability).

Overall, we believe that the development of the interRAI ID represents an important and successful first step toward promoting integrated, comprehensive, and standardized assessment among adults with ID across various provider sectors. Thus, from the perspective of providers, the interRAI ID can permit the development of more individualized life plans based on individual strengths, preferences, and needs, and from a policy perspective, the interRAI ID can present the opportunity to make sounder evidence-based decisions.

\section{REFERENCES}

Aylward, E. H., Burt, D. B., Thorpe, L. U., Lai, F., \& Dalton, A. J. (1995). Diagnosis of dementia in individuals with intellectual disability. Report of the AAMR-IASSID working group for the establishment of criteria for the diagnosis of dementia in individuals with intellectual disability. Washington, DC: American Association on Mental Retardation.

Burrows, A. B., Morris, J. N., Simon, S. E., Hirdes, J. P., \& Phillips, C. (2000). Development of a minimum data set-based depres- sion rating scale for use in nursing homes. Age and Ageing, 29, $165-172$.

Burt, D. B., \& Aylward, E. H. (2000). Test battery for the diagnosis of dementia in individuals with intellectual disability. Working group for the establishment of criteria for the diagnosis of dementia in individuals with intellectual disability. Journal of Intellectual Disability Research, 44, 175-180.

Carmines, E. G., \& Zeller, R. A. (1979). Reliability and validity assessment. Newbury Park, CA: Sage Publications.

Evenhuis, H. M. (1995). Manual of the dementia questionnaire for persons with mental retardation (DMR) (2nd ed.). Zwammerdam, the Netherlands: Hooge Burch, Centre for People with Intellectual Disability.

Hirdes, J. P., Fries, B. F., Morris, J. N., Steel, K., Mor, V., Frijters, D., et al. (1999). Integrated health information systems based on the RAI/MDS series of instruments. Health Care Management Forum, 12, 30-40.

Hirdes, J. P., Martin, L., Curtin-Telegdi, N., Fries, B. E., James, M., Ikegami, N., et al. (2003). interRAI intellectual disabilityPrimer for use of the interRAI-ID. Washington, DC: interRAI.

Martin, L. (2004). Assessing the needs of adults with intellectual disability in different care settings: Development and evaluation of the interRAI-intellectual disability (interRAI-ID). Waterloo, ON: University of Waterloo.

Mor, V. (2004). A comprehensive clinical assessment tool to inform policy and practice: Applications of the minimum data set. Medical Care, 42, 50-59.

Morris, J. N., Fries, B. E., Mehr, D. R., Hawes, C., Phillips, C., Mor, V., et al. (1994). MDS cognitive performance scale. Journal of Gerontology: Medical Sciences, 49, M174-182.

Morris, J. N., Fries, B. E., Steel, K., Ikegami, N., Bernabei, R., Carpenter, G. I., et al. (1997). Comprehensive clinical assessment in community setting: applicability of the MDSHC. Journal of the American Geriatrics Society, 45, 10171024.

Morris, J. N., Fries, B. E., \& Morris, S. A. (1999). Scaling ADLs within the MDS. Journal of Gerontology: Medical Sciences, 54A, M546-M553.

Morris, J. N., Carpenter, I., Berg, K., \& Jones, R. N. (2000). Outcome measure for use with home care clients. Canadian Journal on Aging, 19, 87-105.

Reiss, S. (1994). Reiss screen for maladaptive behavior: Test manual (2nd ed.). Worthington, OH: IDS Publishing Corporation.

Sturmey, P., \& Bertman, L. J. (1995). Validity of the Reiss screen for maladaptive behavior. American Journal on Mental Retardation, 99, 201-206. 